\title{
ANÁLISIS MORFOMÉTRICO Y TECNOLÓGICO DE ARTEFACTOS BIFACIALES. UN CASO DE ESTUDIO DEL GOLFO SAN MATÍAS (PROVINCIA DE RÍO NEGRO, ARGENTINA)
}

\author{
MORPHOLOGICAL AND TECHNOLOGICAL ANALYSES OF \\ BIFACES. A STUDY CASE FROM THE COAST OF SAN MATÍAS \\ GULF (RÍO NEGRO PROVINCE, ARGENTINA)
}

\author{
Jimena Alberti ${ }^{1}$ y Marcelo Cardillo ${ }^{2}$
}

\begin{abstract}
La tecnología bifacial tiene múltiples características vinculadas a la eficiencia. Al ser de tipo conservada permite, entre otras cosas, extender la vida útil de la materia prima y aumentar la cantidad de lascas extraídas por núcleo. Asimismo, los filos bifaciales poseen mayor durabilidad y amplia flexibilidad funcional. En general, el estudio de estos artefactos se basa en el análisis técnico y morfológico, mientras que la variación morfológica propiamente dicha es tratada de manera cualitativa. Sin embargo, esta variación es continua y responde tanto a aspectos funcionales y tecnológicos así como a la historia de vida de estos artefactos. La morfometría geométrica permite caracterizar la variación morfológica tal cual es, es decir, como un continuo. Asimismo, facilita su caracterización y su estudio cuantitativo. En la costa del golfo San Matías esta tecnología está ampliamente representada, con gran variabilidad en las formas y en las materias primas utilizadas. Los resultados del análisis sugieren que no existen diferencias significativas en la manufactura respecto de las diferentes rocas utilizadas: rocas localmente poco abundantes fueron reducidas con la misma intensidad que materias primas más representadas en las fuentes del área. Debido a las características generales de la muestra y a las condiciones ambientales del golfo, consideramos que la manufactura de estos bifaces habría estado dirigida hacia la obtención de herramientas flexibles que habrían permitido la realización de una amplia variedad de tareas.
\end{abstract}

Palabras claves: tecnología bifacial, golfo San Matías, análisis morfométricos, intensidad de reducción, materias primas líticas.

Bifacial technology has many characteristics which are related to efficiency. Because it is a curated technology, it allows, among other things, to extend the use-life of different lithic raw materials and to increase the amount of flakes removed from a core. Moreover, bifacial edges have greater durability and a wide functional flexibility. In general, the study of these artifacts is based on a technical and morphological analysis, while the actual morphological variation is treated qualitatively. However, this variation is continuous and responds to both functional and technological aspects as well as to the life history of these artifacts. The geometric morphometric allows to characterize the morphological variation as it is, a continuum. Moreover, it also facilitates its characterization and its quantitative study. In San Matías Gulf coast this technology is widely represented in the lithic assemblages, with great variability in the final forms and raw materials that were used. The results suggest there are no significant differences regarding the use of different rocks used: raw materials that are locally were reduced to the same extent than raw materials more abundant in the sources of the area. Because of the general characteristics of the sample and environmental conditions of the Gulf, we believe that the manufacture of these bifaces would have been directed towards obtaining flexible tools that would have allowed the performance of a wide variety of tasks.

Key words: Bifacial technology, San Matías Gulf, morphometric analyses, reduction intensity, lithic raw materials.

Un instrumento lítico debe ser capaz de resolver las contingencias producidas por las diferencias espaciales y temporales entre la localización de la fuente de materias primas y la del recurso explotado (Kelly 1988). Esto implica que posea, entre otras, algunas propiedades deseables vinculadas al diseño de los artefactos, como la flexibilidad y versatilidad. Es decir, la capacidad de responder de manera eficiente a distintas funciones con o sin necesidad de ser modificado previamente (discusión en Nelson 1991). Al respecto, la tecnología bifacial tiene la ventaja de satisfacer diversos requerimientos

1 Instituto Multidisciplinario de Historia y Ciencias Humanas (IMHICIHU) - Consejo Nacional de Investigaciones Científicas y Técnicas (CONICET), Saavedra 15, 5to. piso. CP (1083) - Buenos Aires, Argentina. jimealberti@ gmail.com

2 Instituto Multidisciplinario de Historia y Ciencias Humanas (IMHICIHU) - Consejo Nacional de Investigaciones Científicas y Técnicas (CONICET), Universidad de Buenos Aires, Buenos Aires, Argentina. marcelo.cardillo@gmail.com.

Recibido: octubre 2014. Aceptado: octubre 2015. 
funcionales y de performance y siguiendo a Kelly (1988) puede funcionar de tres formas diferentes, de acuerdo con las circunstancias bajo las cuales se lo utilice: como núcleo, como herramienta en sí o como un subproducto de la talla.

En el presente trabajo se realiza un primer acercamiento a la variabilidad en diseño y materia prima de los artefactos bifaciales recuperados en diferentes muestreos realizados en las localidades arqueológicas de la costa rionegrina del golfo San Matías. A partir de los análisis efectuados se determinaron los tipos de rocas utilizadas para la manufactura de estos artefactos y la variabilidad de forma y tamaño existente dentro del grupo de los artefactos manufacturados mediante la talla bifacial.

\section{La Tecnología Bifacial}

De acuerdo con Kelly (1988), en general los bifaces son herramientas conservadas, ya que su manufactura requiere de una alta inversión de energía y suelen formar parte de un sistema tecnológico cuya característica principal es la confiabilidad (Bleed 1986). Como se mencionó, estos pueden cumplir tres roles diferentes (Kelly 1988). El primero de ellos, el de funcionar como núcleos, implica que los bifaces sirven como fuentes de materia prima inmediatamente disponible, ya que permiten la extracción de lascas finas y afiladas que pueden ser formatizadas en diferentes tipos de instrumentos. Contar con un núcleo bifacial permite extraer lascas con mayor superficie de bordes -aristas-aprovechables (comparado con otro tipo de núcleos), al tiempo que se minimiza la cantidad de roca transportada. Esta función de los bifaces como núcleos se espera que incremente en relación con la escasez de materia prima, en partidas logísticas para disminuir la cantidad de peso cargado, cuando se desconoce la disponibilidad de rocas aptas para la talla o condiciones de incertidumbre (Kelly 1988).

El segundo posible uso de los bifaces es como herramientas, ya que pueden ser usados en una multiplicidad de tareas y proveen mayor cantidad de bordes disponibles para cortar con un menor desgaste que los de una lasca sin modificar, ya que son más robustos (Kelly 1988; también Bleed 1986; Nelson 1991).

Por último, como subproducto de la manufactura, los bifaces son el resultado de un sistema de reducción particular que apunta a darle gran importancia al enmangue del artefacto (Kelly 1988). En este caso, los bifaces pueden aparecer más mantenidos y su reemplazo se dará generalmente en los sitios residenciales, donde probablemente se almacene el material orgánico necesario para el enmangue (Kelly 1988).

De acuerdo con Aschero y Hocsman (2004), los bifaces tienen la particularidad de ser una forma potencialmente modificable en caso de encontrarse con situaciones cambiantes que requieran la manufactura de un instrumento diferente. En términos de Nelson (1991), en estos se priorizaría la mantenibilidad como variable de diseño, con sus dos opciones posibles: la flexibilidad y la versatilidad. Que un artefacto sea mantenible implica que puede ser usado en una variedad de circunstancias a través del reemplazo de partes o de cambios en la forma de uso. A su vez, la flexibilidad permite la reformatización de la herramienta dada para cumplir con diferentes requerimientos, mientras que la versatilidad implica que esta tiene una forma generalizada tal que sirve para ser usada en una multiplicidad de tareas (Nelson 1991). A su vez, los bifaces tienen la particularidad de que son transportables, es decir, que pueden ser llevados hacia el lugar donde se realizará la tarea (Nelson 1991). De acuerdo con Kuhn (1994), el tamaño y contenido de los toolkits variará de acuerdo con factores como el ambiente y la forma de transporte. El equipamiento que los individuos llevan con ellos debe ser capaz de resolver una amplia variedad de circunstancias y debe durar hasta que se lo pueda reemplazar, y los bifaces cumplen, potencialmente, con estos requisitos.

Teniendo en cuenta estas características de los artefactos bifaciales, en este trabajo se presentan por primera vez los resultados de los análisis morfométricos y tecnológicos de los artefactos bifaciales recuperados en la costa rionegrina del golfo San Matías con el fin de determinar el papel que los mismos habrían cumplido dentro de la tecnología manufacturada y utilizada por los grupos cazadores-recolectores que habitaron el área durante el Holoceno Medio y Tardío.

\section{El Área de Estudio}

De acuerdo con sus características geológicas y geomorfológicas, la costa rionegrina del golfo San Matías se divide en dos sectores, norte y oeste. El primero de ellos corre de oeste a este entre la ciudad de San Antonio Oeste y la desembocadura 
del río Negro en el océano Atlántico (Figura 1), y en él se alternan sectores con acantilados abruptos con playas y entrantes costeros. El segundo, en cambio, corre de norte a sur, entre Las Grutas y el límite con la provincia de Chubut (Figura 1) y su rasgo principal es la presencia de la meseta de Somuncurá, una planicie estructural lávica que desciende hacia el mar en forma de pedimentos de flanco (González Díaz y Malagnino 1984).

Respecto de la distribución de recursos, la costa oeste es ambientalmente más homogénea que la parte norte del golfo, y presenta una menor diversidad de especies marinas, así como escasez de agua dulce y de reparos topográficos (Favier

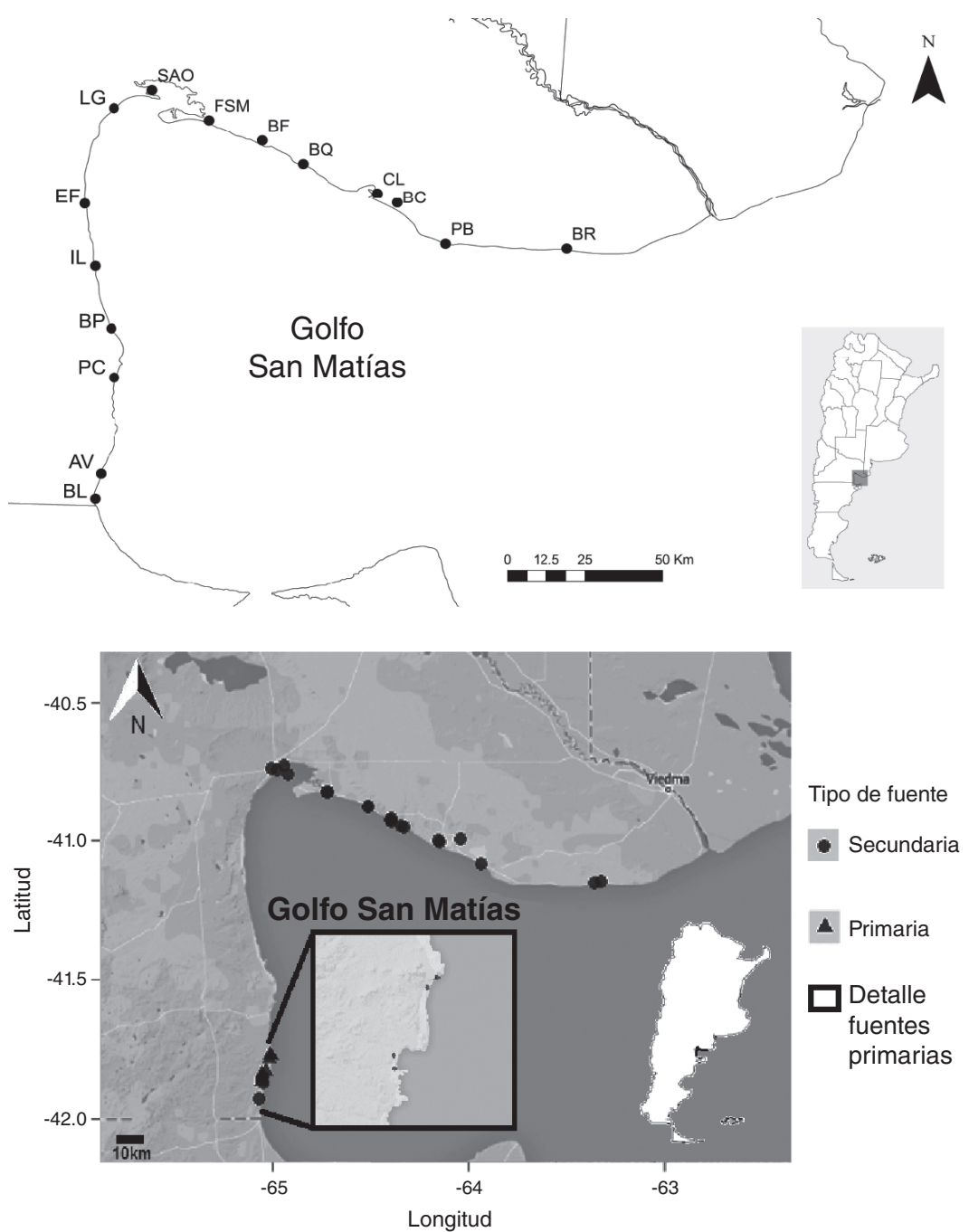

Figura 1. Costa rionegrina del golfo San Matías. En la figura superior se marcan las localidades principales de las que proviene la muestra analizada; en la inferior, las ubicaciones de las fuentes de materias primas líticas. BR: Bahía Rosas. PB: Promontorio Belén. BC: Bahía Creek. CL: Caleta de los Loros. BQ: Bajo de la Quinta. BF: Bahía Final. FSM: Faro San Matías. SAO: San Antonio Oeste. LG: Las Grutas. EF: El Fuerte. IL: Islote Lobos. BP: Bahía Pozos. PC: Punta Colorada. AV: Arroyo Verde. BL: Bahía Lobos.

Coast of San Matías Gulf, Río Negro province. In the upper figure the main archaeological localities from which the analyzed sample comes from are shown; in the bottom figure the locations of the lithic raw materials sources are detailed. BR: Bahía Rosas. PB: Promontorio Belén. BC: Bahía Creek. $C L$ : Caleta de los Loros. BQ: Bajo de la Quinta. BF: Bahía Final. FSM: Faro San Matías. SAO: San Antonio Oeste. LG: Las Grutas. EF: El Fuerte. IL: Islote Lobos. BP: Bahía Pozos. PC: Punta Colorada. AV: Arroyo Verde. BL: Bahía Lobos. 
Dubois y Borella 2011). Estas características junto con la baja señal arqueológica y la presencia de obsidiana proveniente de lugares distantes (Favier Dubois, Stern y Cardillo 2009), han dado lugar a la propuesta de que esta área habría sido utilizada como un espacio de circulación. Este sector habría sido ocupado de forma menos intensiva, aunque presentando ciertos lugares especialmente atractivos para la ocupación humana (Borella et al. 2007; Favier Dubois y Borella 2011) hacia el sur. Los loci localizados en esta parte de la costa del golfo, alrededor de 42 en total, han sido datados entre los 3.200 y los 700 años ${ }^{14} \mathrm{C}$ a.p. (Borella et al. 2015; Favier Dubois y Borella 2011).

Por otro lado, la costa norte del golfo posee una bioproductividad mayor, ya que se combinan en esta área la presencia de agua dulce en dunas, la accesibilidad a las especies marinas, y la existencia de reparos topográficos (Borella 2006; Favier Dubois y Borella 2011). Esta área en general fue más intensa y redundantemente ocupada que la parte oeste del golfo. Las fechas de los loci estudiados hasta el momento se ubican entre los ca. $6.000 \mathrm{y}$ los 450 años ${ }^{14} \mathrm{C}$ a.p. (Favier Dubois, Borella y Tykot 2009). Para esta costa se ha propuesto un modelo de consumo de los recursos marinos de tres etapas basado en los análisis isotópicos sobre restos esqueletales humanos, en donde se sostiene que las poblaciones humanas habrían girado de una dieta predominantemente marina en los inicios de la ocupación, con una tecnología de tipo expeditiva orientada hacia la obtención, procesamiento y consumo de estos recursos, hacia una dieta mixta con un componente terrestre más intenso para los momentos más tardíos, y un cambio hacia la aparición de puntas de proyectil más pequeñas que las de momentos tempranos (Favier Dubois, Borella y Tykot 2009).

Respecto de las fuentes de materias primas, en la costa norte estas son principalmente de tipo secundario, distribuidas de forma abundante y ubicua en el ambiente (Alberti 2012; Favier Dubois y Alberti 2014) (Figura 1). Dentro de estos depósitos predominan las rocas volcánicas intermedias-básicas de calidad regular a muy buena para la talla, mientras que rocas silíceas y calcedonias son más escasas y de calidad muy buena a excelente (Favier Dubois y Alberti 2014). En muestreos realizados de las fuentes en esta área, de 1.306 nódulos se recuperó $67 \%$ de nódulos de rocas volcánicas y solo $25 \%$ de sílex y calcedonia (Alberti 2015). La forma predominante de estos nódulos es prolada, con un largo promedio de $60 \mathrm{~mm}$. Por lo demás, en la costa oeste las fuentes secundarias conformadas por cordones litorales son menos abundantes (Figura 1). Las rocas silíceas y las calcedonias, en cambio, aparecen en mayor frecuencia en esta porción de la costa (55\% de 339 nódulos muestreados), aunque en densidades menores a las observadas en la costa norte en relación con las rocas volcánicas intermedias-básicas (18\%) (Alberti 2015; Alberti y Cardillo 2015). A diferencia de lo que sucede en la costa norte, en esta costa la forma predominante de los nódulos es tabular, con un largo promedio de $58 \mathrm{~mm}$. Creemos que esta variabilidad en el tipo, densidad y distribución de los recursos líticos puede afectar el modo de producción de instrumentos bifaciales, como se verá más adelante.

\section{Materiales y Métodos}

La definición de bifaz que se siguió como criterio para delimitar la muestra es la presentada por Kelly (1988), en donde un artefacto bifacial es una lasca o un núcleo que ha sido reducido mediante la percusión o la presión, en ambas caras desde dos ejes opuestos y paralelos, y al que se le ha dado una forma particular. Entendemos en este trabajo a los bifaces en términos morfológicos y no necesariamente funcionales, ya que sin un análisis de orden funcional no es posible determinar si un artefacto bifacial dado fue utilizado como instrumento o si, simplemente, sirvió como núcleo o forma base. La muestra analizada está integrada por 126 artefactos, enteros $(\mathrm{n}=50)$ y fragmentados $(n=76)$, confeccionados con diferentes materias primas (Tabla 1) (para algunos ejemplos Figura 2), seleccionados a partir de un conjunto mayor integrado por diferentes clases artefactuales. Dentro de este subconjunto se diferenciaron cuatro subconjuntos: preformas bifaciales, preformas de cabezales líticos, bifaces y cabezales líticos.

Para el análisis tecnomorfológico de las piezas se siguieron los criterios establecidos en Aschero (1983 [1975]). Este tipo de análisis permite el abordaje de los artefactos para clasificarlos de acuerdo con sus atributos de forma. Mientras que el análisis tecnológico permite identificar los rasgos que entran en juego en el diseño y manufactura de los artefactos (p.ej., tipo de materia prima utilizada), el análisis morfológico evalúa la forma del artefacto de manera descriptiva, teniendo en cuenta la representación 
Tabla 1. Distribución de tipos de artefactos por materia prima. Type of artifacts divided according to type of raw material type.

\begin{tabular}{lrrrrr}
\hline Materia prima & $\begin{array}{c}\text { Preforma } \\
\text { bifacial }\end{array}$ & $\begin{array}{c}\text { Preforma } \\
\text { cabezal } \\
\text { lítico }\end{array}$ & Bifaz & $\begin{array}{c}\text { Cabezal } \\
\text { lítico }\end{array}$ & $\mathrm{N}$ \\
\hline Brecha silicificada & 0 & 0 & 0 & 1 & 1 \\
Calcedonia & 4 & 6 & 4 & 23 & 37 \\
Chert biogénico & 3 & 0 & 1 & 1 & 5 \\
Jaspe & 0 & 0 & 0 & 3 & 3 \\
Obsidiana & 0 & 0 & 0 & 5 & 5 \\
Ópalo & 0 & 0 & 1 & 4 & 5 \\
Sílex & 15 & 4 & 7 & 27 & 53 \\
Toba & 0 & 0 & 0 & 1 & 1 \\
Toba silicificada & 0 & 0 & 0 & 2 & 2 \\
Volcanita ácida & 0 & 1 & 0 & 2 & 3 \\
Volcanita ácida silicificada & 0 & 1 & 0 & 0 & 1 \\
Volcanita intermedia-básica & 2 & 0 & 1 & 7 & 10 \\
N & 24 & 12 & 14 & 76 & 126 \\
\hline
\end{tabular}

gráfica del mismo, el uso de escalas y la generación de patrones que permitan la identificación clara de artefacto en sí.

El análisis macroscópico de las materias primas se realizó de acuerdo con Alberti y Fernández (2015) y las rocas fueron luego comparadas con las que integran la litoteca de referencia del área para obtener una identificación más exacta de los tipos litológicos.

Para determinar la clase técnica (sensu Aschero y Hocsman 2004) de cada pieza, la que distingue grados de trabajo invertidos en la manufactura de cada artefacto a partir de la superposición de lascados en las caras, se midió la cantidad de lascados en ambas caras de los artefactos y la cantidad de ellos que llegan hasta el centro y/o lo sobrepasan. De acuerdo con Hocsman y Escola (2006-2007), entendemos
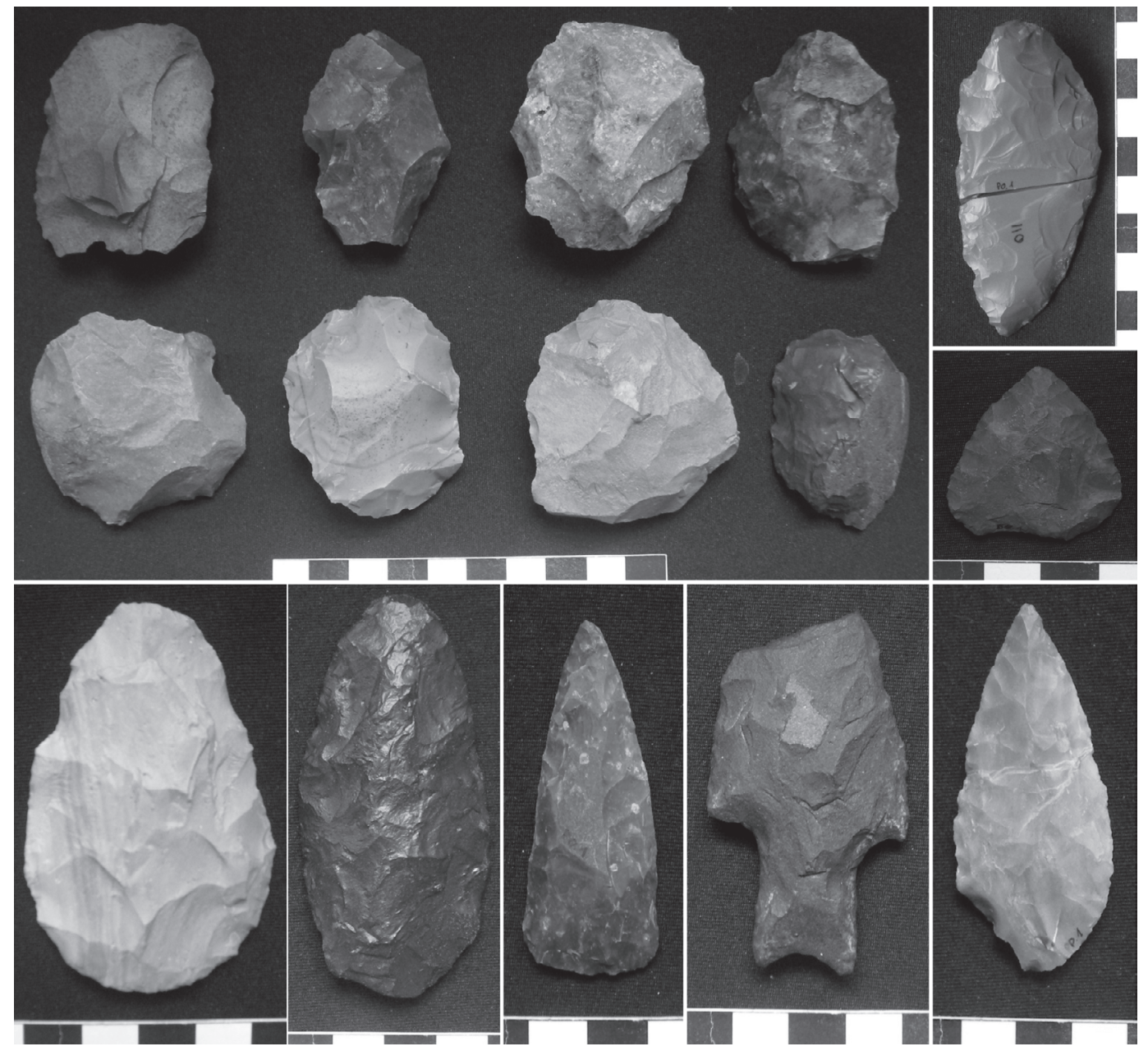

Figura 2. Bifaces y puntas de proyectil integrantes de la muestra analizada.

Bifaces and projectile points of the sample. 
como reducción bifacial a artefactos con lascados bifaciales que no cubren el centro de la cara o, de hacerlo, no se superponen con los que provienen del otro borde. Además, el adelgazamiento bifacial implica piezas en las que la intención estuvo puesta en rebajar el espesor sin afectar el ancho, de forma tal de generar una sección generalmente biconvexa con lascados que afectan más las caras que los bordes (Hocsman y Escola 2006-2007).

Para definir el grado de reducción del bifaz se utilizaron variables definidas por Nami (1988): el grado de regularidad del contorno y la presencia de simetría o no de las piezas, vistas en norma frontal. Las piezas con aristas asimétricas, contorno irregular y ausencia de retoques de regularización en sus filos fueron consideradas bifaces o preformas, mientras que las que cumplían estos requisitos, puntas de proyectil. Además se registró el peso y las medidas máximas de cada pieza (ancho, largo y espesor). Todas las variables métricas fueron tomadas con calibre y balanza digital, y volcadas en una planilla Excel confeccionada para tal fin. Las piezas enteras fueron calcadas, de forma tal de poder realizar los análisis morfométricos correspondientes.

Con el fin de determinar si existen diferencias significativas entre los distintos productos de la talla (bifaces, preformas de cabezales líticos y/o sus preformas) y la selección de materias primas, así como su relación con la simetría general de los artefactos y de sus aristas se realizaron test de $\mathrm{Chi}^{2}$, comúnmente utilizados en la comparación de variables categóricas. En todos los casos, debido a la baja frecuencia de algunas categorías (menores a cinco), se utilizó la estimación de Monte Carlo para determinar la probabilidad del test (Fishman 1995).

\section{Análisis de la forma}

Los análisis de forma fueron realizados únicamente sobre las piezas enteras del conjunto $(\mathrm{n}=50)$. Para ello se empleó el análisis de Fourier Elíptico (EFA) (Kuhl y Giardina 1982). El mismo es especialmente útil para lograr una descripción cuantitativa de la forma, la que puede ser empleada para describir o comparar conjuntos de objetos a partir de sus contornos. Mediante este método, la descripción de la forma del contorno se realiza a partir de $n$ parámetros denominados harmónicas, cada una de las cuales contribuye a la descripción de la forma empírica en diferente grado. Las primeras harmónicas describen un contorno de una manera muy general, mientras que las últimas describen variaciones que ocurren a pequeña escala, de forma tal que en conjunto representan todas las posibles dimensiones de la variación. Las primeras harmónicas capturan la forma general del contorno (redondeamiento, alargamiento, compresión), mientras que las de alto orden muestran la angularidad del mismo, como puede ser por ejemplo la irregularidad de las aristas de los artefactos. Posteriormente, las harmónicas son normalizadas para eliminar el efecto del tamaño, orientación y rotación. El grado de resolución necesario para la descripción de una forma cualquiera puede ser manipulado de esta manera a partir de la selección del número de harmónicas. La utilización de esta metodología tiene como principal ventaja que permite realizar una descripción cuantitativa de la forma de manera continua. Esto disminuye, entre otras cosas, la subjetividad de la definición tipológica o en categorías de forma cuya variación se comporta muchas veces como un continuo (Gero y Mazzullo 1984), además de permitir su tratamiento estadístico. Para la digitalización de los contornos se emplearon calcos de los mismos realizados sobre papel, lo que permite una mínima deformación del contorno y facilita la digitalización en perfiles básicamente bidimensionales. Estos calcos fueron digitalizados a 300 dpi en escáner plano. En cada contorno se distribuyeron 100 puntos equidistantes a partir del programa TPSdig2 (Rohlf 2006). Estos puntos fueron la base para la estimación de las harmónicas. Los archivos de puntos fueron procesados mediante el programa R (R Development Core Team 2009), en donde se realizó previamente la estandarización de los contornos a base de la primer harmónica. Esta estandarización remueve la información relativa al tamaño, posición y rotación de cada ejemplar, tal que se preserva solo la información relativa a la forma. La cantidad de harmónicas mínimas necesarias para describir correctamente el contorno fue determinada mediante la estimación de potencia sobre la cantidad de varianza explicada por las harmónicas en relación con el total de la variación (Kuhl y Giardina 1982; Rohlf 2006). Esto permite tomar el número mínimo de descriptores y dejar de lado pequeñas variaciones relativas a errores aleatorios producidos durante la copia o digitalización de los contornos.

Posteriormente las harmónicas fueron sometidas a un análisis de componentes principales, con el fin de obtener las tendencias generales de variación morfológica en la muestra. Estas coordenadas principales fueron empleadas en análisis descriptivos 
y comparativos junto con las demás variables categóricas y continuas con el fin de explorar la relación entre aspectos tecnológicos y morfológicos.

Por último, las variables morfológicas fueron utilizadas en análisis de regresión por mínimos cuadrados con el fin de determinar la existencia de una relación de dependencia entre la forma y variables métricas vinculadas a procesos de formatización/reducción de las formas base, como promedio de lascados, la proporción de corteza en ambas caras y el peso del artefacto medido en gramos. Con el fin de ajustar las variables al requisito de homogeneidad de las varianzas (homocedasticidad) para una mayor adecuación al modelo lineal, las variables fueron transformadas a su logaritmo natural en el caso que fue necesario. Posteriormente se estimó el coeficiente de determinación $\mathrm{R}^{2}$ y se realizó el test de homocedasticidad de Breuch-Pagan para determinar el cumplimiento del supuesto de homocedasticidad de los residuos del modelo. Estos análisis fueron realizados mediante el programa Past 3.0 (Hammer et al. 2001).

\section{Resultados}

El primer paso para abordar el análisis de la muestra fue clasificar los diferentes artefactos: si eran preformas o artefactos terminados y por materia prima. Los resultados se presentan en la Tabla 1.

Para abordar la existencia de simetría en los artefactos de acuerdo con la materia prima utilizada para elaborarlos y la presencia de aristas regulares o irregulares, se dividió el conjunto de piezas de acuerdo con los distintos tipos de rocas, como se presenta en la Tabla 2.

Para determinar si las diferencias registradas en la frecuencia de bifaces simétricos y asimétricos y con arista regular o irregular entre las materias eran estadísticamente significativas, se realizaron test de $\mathrm{Chi}^{2}$; el mismo test se realizó también tomando en cuenta solo las rocas más abundantes de la muestra (calcedonia y sílex). En ambos casos no se contrastó la $\mathrm{H}_{0}$ de independencia del test, lo que sugiere que no existirían diferencias en la regularidad de las aristas en relación con el tipo de roca empleado.

En cuanto a la simetría relativa, tampoco se detectaron diferencias vinculadas a las materias primas empleadas, es decir, no hay una tendencia significativa hacia la manufactura de bifaces más o
Tabla 2. Cantidad de artefactos simétricos y asimétricos, y con arista regular o irregular divididos por tipo de materia prima.

Number of symmetric and asymmetric artifacts, and with regular or irregular edge divided according to type of raw material type.

\begin{tabular}{lrrrr}
\hline \multirow{2}{*}{ Materia prima } & \multicolumn{2}{c}{ Simetría } & \multicolumn{2}{c}{ Morfología arista } \\
\cline { 2 - 5 } & Si & No & Regular & Irregular \\
\hline Brecha silicificada & 1 & 0 & 1 & 0 \\
Calcedonia & 27 & 10 & 26 & 11 \\
Chert biogénico & 3 & 2 & 3 & 2 \\
Jaspe & 3 & 0 & 3 & 0 \\
Obsidiana & 3 & 2 & 5 & 0 \\
Ópalo & 5 & 0 & 5 & 0 \\
Sílex & 41 & 12 & 32 & 21 \\
Toba & 1 & 0 & 1 & 0 \\
Toba silicificada & 2 & 0 & 2 & 0 \\
Volcanita ácida & 3 & 0 & 2 & 1 \\
Volcanita ácida silicificada & 1 & 0 & 1 & 0 \\
Volcanita intermedia-básica & 8 & 2 & 8 & 2 \\
N & 98 & 28 & 89 & 37
\end{tabular}

menos simétricos o con arista regular dependiendo de la materia prima utilizada. En el caso de la simetría, el test dio un estadístico de $\mathrm{Chi}^{2}=6,92 ; \mathrm{p}>0,05$ (todos los tipos de roca), y $\mathrm{Chi}^{2}=0,22 ; \mathrm{p}>0,05$ para el caso de las calcedonias y el sílex.

En el caso de la regularidad de la arista el valor del estadístico es $\mathrm{Chi}^{2}=10,89 ; \mathrm{p}>0,05$ para el total de rocas, $\mathrm{y} \mathrm{Chi}^{2}=0,93$ con un $\mathrm{p}>0,05$ para las calcedonias y el sílex. Asimismo se realizó un test sobre los distintos productos de la talla: cabezales líticos, bifaces y sus respectivas preformas, en donde tampoco se pudo contrastar la hipótesis nula de la no diferencia entre las materias primas empleadas en las distintas categorías $\left(\mathrm{Chi}^{2}=22,17 ; \mathrm{p}>0,05\right)$ y $\mathrm{Chi}^{2}=6,824 ; \mathrm{p}>0,05$ (solo para calcedonia y sílex). Esto sugiere que no existe una preferencia clara en el empleo de alguna materia prima en particular para la manufactura de determinados artefactos, al menos dentro de las categorías de rocas estudiadas. Esto sugiere que las tendencias observadas en un mayor número de categorías presentes en rocas criptocristalinas puede deberse simplemente a factores aleatorios, como el tamaño de la muestra.

Esta falta de preferencia de cierto tipo de rocas para la manufactura de bifaces detectada para el caso de la calcedonia y el sílex también se ha verificado para todos los tipos de rocas en otro tipo de instrumentos, como son, por ejemplo, los manufacturados a través de la talla unifacial. La 
calcedonia y el sílex son las rocas más utilizadas en ambas costas del golfo San Matías para todo el lapso que duró la ocupación del área, manufacturándose con estas rocas todo tipo de instrumentos (Alberti 2013, 2015). Por el contrario, las rocas volcánicas intermedias-básicas y ácidas han sido utilizadas preferencialmente para la manufactura de artefactos unifaciales, posiblemente debido a su menor calidad para la talla. Es de destacar, entonces, que aunque la calcedonia y el sílex presentan en general mejor calidad para la talla, este tipo de rocas fueron usadas también para la talla de artefactos unifaciales y con filos simples (Alberti 2015).

Para determinar la intensidad de reducción de los artefactos se cuantificó la cantidad de negativos de lascados en cada una de las caras de las piezas y la cantidad de ellos que llegaban hasta el centro o lo sobrepasaban. Para describir estos resultados se tomaron estadísticos descriptivos de la muestra.
Así, la media de negativos en la cara A de las piezas es de 14 negativos (mínimo 2, máximo 56, desvío estándar 9,4), mientras que en la cara B es de 14 también, con un mínimo de 3 y un máximo de 45 por pieza (desvío estándar 8,5). Sin embargo, la media del número de negativos que llegan hasta el centro en las piezas es 2 , con un mínimo de 0 y un máximo de 9 negativos por pieza (desvío estándar 0,18 ). Esto da cuenta de que el adelgazamiento bifacial no es la técnica predominante en estos conjuntos, sino que, por el contrario, predomina la reducción bifacial (ver supra).

\section{Análisis morfométrico}

Los resultados del análisis morfométrico muestran las tendencias generales de variación morfológica en las muestras (Figuras 3 y 4). El primer componente, que explica el $60 \%$ de la

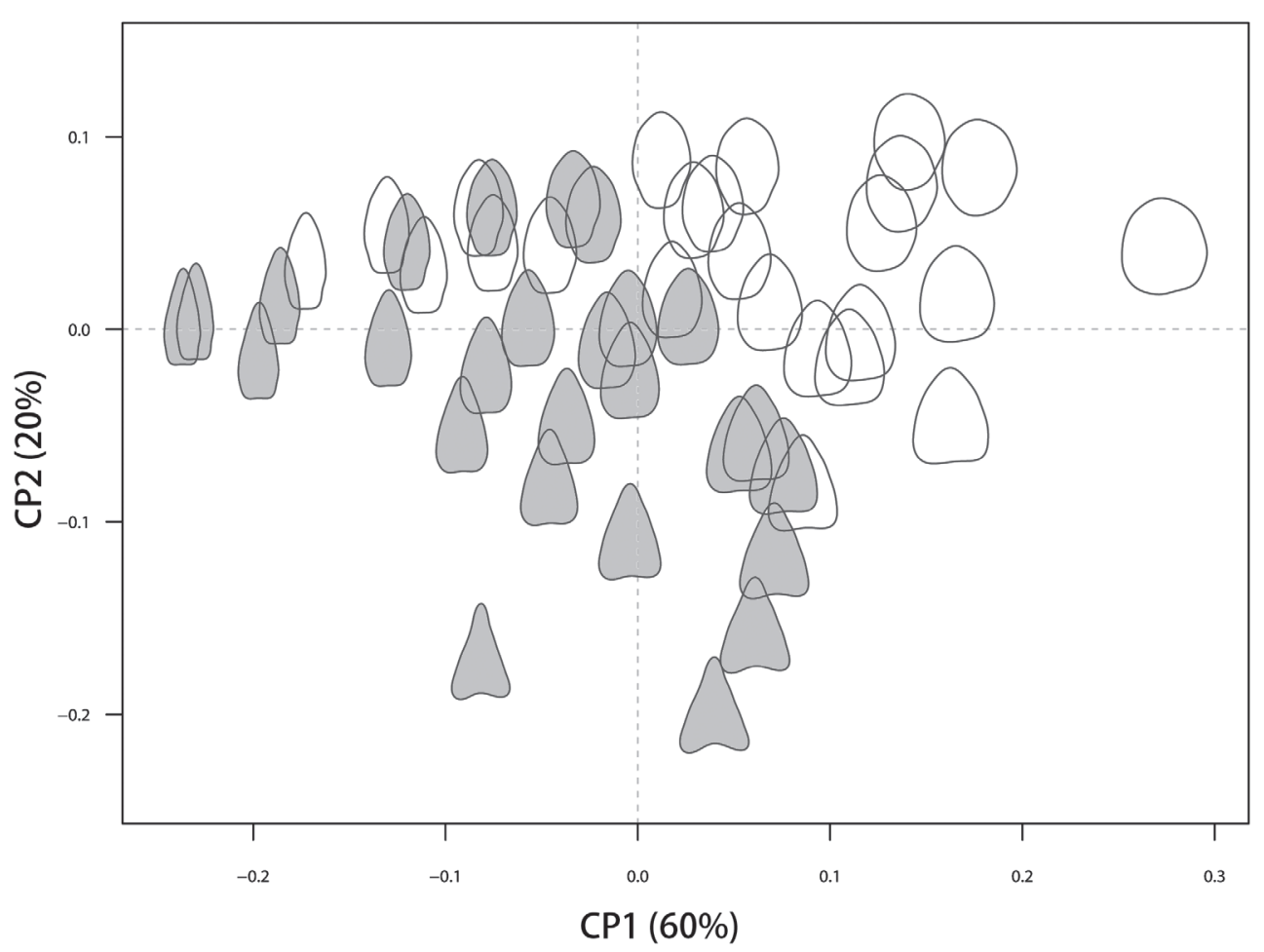

Figura 3. Componentes principales de variación morfológica en la muestra analizada. Los contornos reconstruidos representan hasta una desviación estándar de la forma media. Formas grises: cabezales líticos. Formas blancas: bifaces y preformas bifaciales. CP1 elongación-redondeamiento relativo, CP2 forma de la base cóncava-convexa/saliente. Main components of morphological variation in the sample. The reconstructed contours represent a standard deviation of the mean shape. Gray shapes: projectile point. White shapes: bifaces and bifacial preforms. CP1 relative elongation-roundness, $\mathrm{CP} 2$ concave-convex/stemming base shape. 


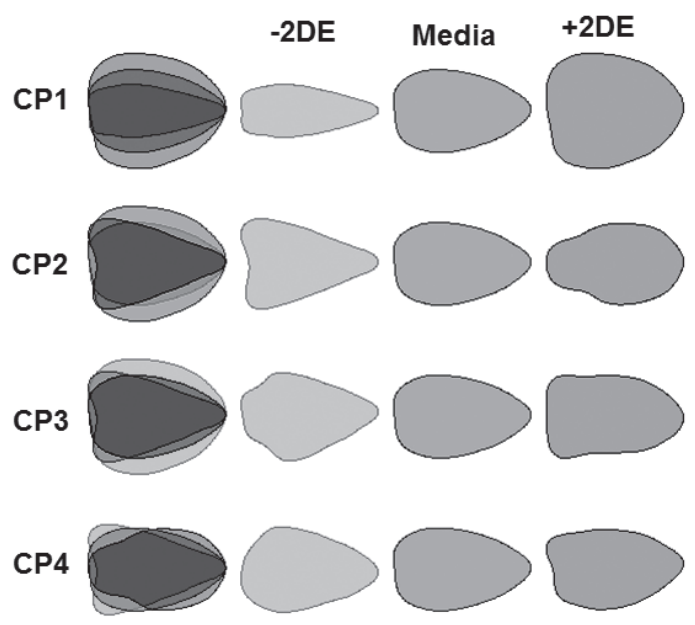

Figura 4. Reconstrucción de la variación en torno a la media (dos desvíos estándar) para cuatro componentes principales de forma.

Reconstruction of the variation around the mean (two standard deviations) for four main form components.

variación total, señala la tendencia general de cambio entre formas de limbo comprimido y alargado y otras de contorno redondeado u oval. El segundo componente explica el $20 \%$ de la variación total y describe el cambio entre formas con pedúnculo destacado o esbozado y contornos apedunculados. Los dos componentes siguientes (Figura 4) describen variación más loca $\Omega$ lizada, así como la asimetría de los artefactos, particularmente en la parte mediainferior del limbo.

La distribución a lo largo del primer componente es relativamente homogénea, observándose una distribución de casos prácticamente continua a lo largo de este eje. El segundo componente, en cambio, muestra mayor dispersión, especialmente en relación con las formas triangulares. Asimismo, se observa que existe un escaso solapamiento entre preformas, bifaces y cabezales líticos, ya que tienden a ocupar distintos sectores del espacio morfológico. Para explorar las relaciones entre variación métrica y forma utilizamos los dos primeros componentes simétricos que explica la mayor parte de la varianza total.

Al correlacionar las variables métricas con las coordenadas de forma se observó una relación significativa entre el peso $\mathrm{R}^{2}=0,47 ; \mathrm{p}<0,01$ (BreuschPagan homocedasticidad 8,50, $\mathrm{p}=0,003$ ) y el promedio de lascados $\mathrm{R}^{2}=0,37 ; \mathrm{p}<0,001$ (BreuschPagan homocedasticidad 0,17; $\mathrm{p}=0,67)$. Si bien las variables independientes fueron transformadas a logaritmo para ajustarlas a los requerimientos del modelo de regresión, los residuos en el primer caso son heterocedásticos, lo que sugiere una dispersión significativa de variables al menos en algunos sectores de la recta (Figura 5). Esta variabilidad se observa principalmente al inicio de la pendiente en donde hay mucha variabilidad morfológica en torno al peso. Sin embargo, se observa en este caso una tendencia general a que las morfologías más pesadas sean más redondeadas u ovales en torno a los $20 \mathrm{~g}$, lo que coincide con los bifaces y las preformas (Figura 5A). En el segundo caso, el modelo de regresión señala una tendencia al adelgazamiento y compresión de la morfología a medida que se incrementa el número de lascados y en torno a un promedio de 11 lascados se observa una transición entre preformas-bifaces y cabezales líticos (Figura 5B).

\section{Forma y Materias Primas}

La distribución de materias primas en relación con los dos primeros ejes del espacio morfológico muestra un amplio solapamiento que sugiere que 
no puede determinarse una relación clara entre una materia prima determinada y variantes morfológicas. Esto último es concordante con los resultados del test de independencia de $\mathrm{Chi}^{2}$ sobre el total de la muestra. Sin embargo sí se observan diferencias en la dispersión de las distintas categorías. Esto se da en particular en el grupo de las rocas volcánicas intermedias-básicas, estas parecen tener una variabilidad morfológica menor (Figura 6), pese a tener el mismo peso y cantidad promedio de lascados que los artefactos manufacturados sobre calcedonia. En este sentido, cabe destacar que en los muestreos de materias primas realizados en la costa norte del golfo (Favier Dubois y Alberti 2014) el peso de los nódulos fue uno de los factores registrados. Así, los nódulos de rocas volcánicas básicas $(n=75)$ pesan un promedio de $74 \mathrm{~g}$, con un rango que va entre 2 y $422 \mathrm{~g}$ y un desvío estándar de 88,2 , mientras que los de calcedonia $(n=83)$ pesan $86 \mathrm{~g}$, con un rango que se ubica entre 2 y $194 \mathrm{~g}$, con un desvío estándar de 43,4 (Alberti 2015). De esta forma, se puede afirmar que aunque el producto final de la manufactura de bifaces y/o cabezales líticos y sus respectivas preformas sea similar en cuanto al peso, se habría partido de nódulos más pesados en el caso de las calcedonias. Este hecho podría llevar a pensar en estrategias de reducción más intensivas para esta última materia prima, aunque el número de lascados en ambas caras de los productos terminados tanto de rocas volcánicas básicas como de calcedonias sea similar.

\section{Discusión y Conclusiones}

Los análisis realizados en las distintas categorías de bifaces reconocidas y los diversos tipos de materias primas sugieren que estas últimas habrían sido utilizadas indistintamente para la confección de los diferentes productos de la talla bifacial. Este patrón es consistente sobre la totalidad de la muestra (incluyendo rocas que se encuentran en muy baja frecuencia) o considerando solamente las más comunes, particularmente la calcedonia y el sílex. Por otro lado su mayor abundancia en relación con este tipo de artefactos no está vinculada a la disponibilidad de estas rocas en el área, ya que están presentes en muy baja frecuencia dentro de la oferta litológica de los depósitos secundarios (Alberti 2015). Considerando la disponibilidad de rocas en el espacio, sería de esperar que calcedonias y sílex hubiesen sido aprovechadas más intensivamente y de forma distinta que, por ejemplo, las volcanitas intermedias-básicas que constituyen el grupo más abundante en las fuentes de materias primas (Favier Dubois y Alberti 2014). Sin embargo, este
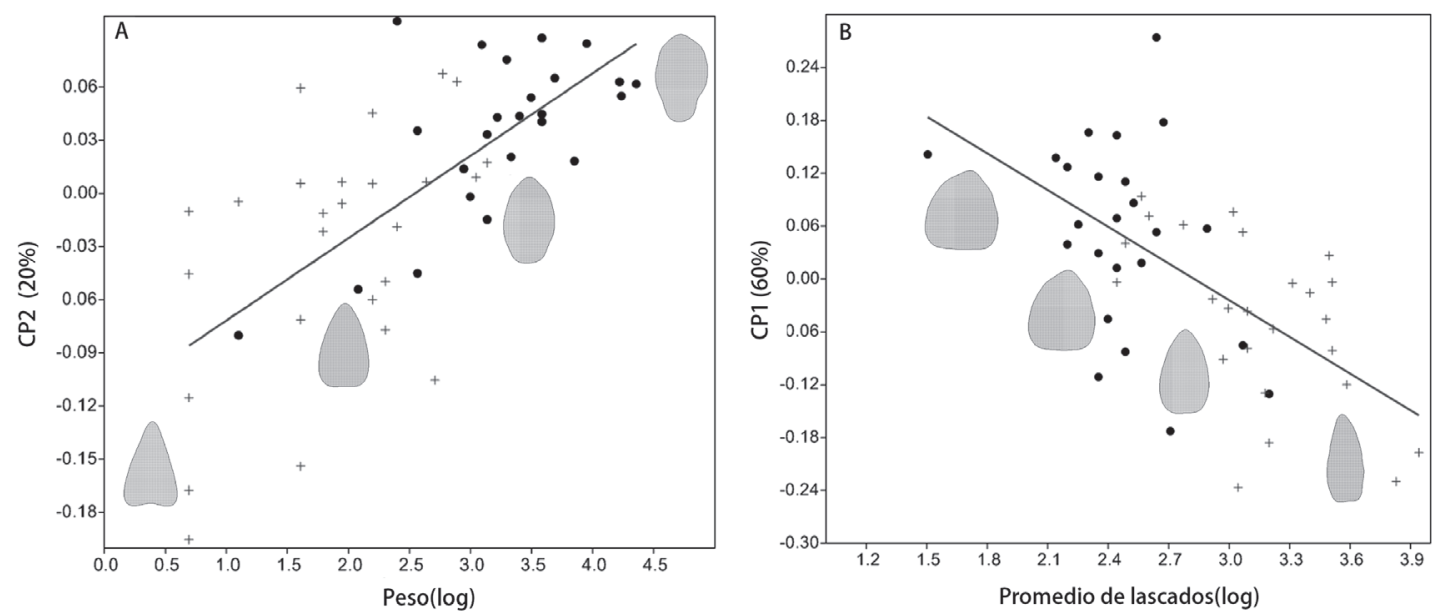

Figura 5. A. Regresión entre el logaritmo del peso y el segundo componente. B. Regresión entre el logaritmo del promedio de lascados y el primer componente. Los puntos corresponden a las preformas y los bifaces y las cruces a los cabezales líticos. CP1: redondez de los limbos. CP2: presencia y ausencia de pedúnculo.

A. Regression between the logarithm of the weight and the second component. B. Regression between the logarithm of the flake scars average and the first component. The points show preforms and bifaces and crosses show projectile points. CP1: roundness. CP2: stemming. 


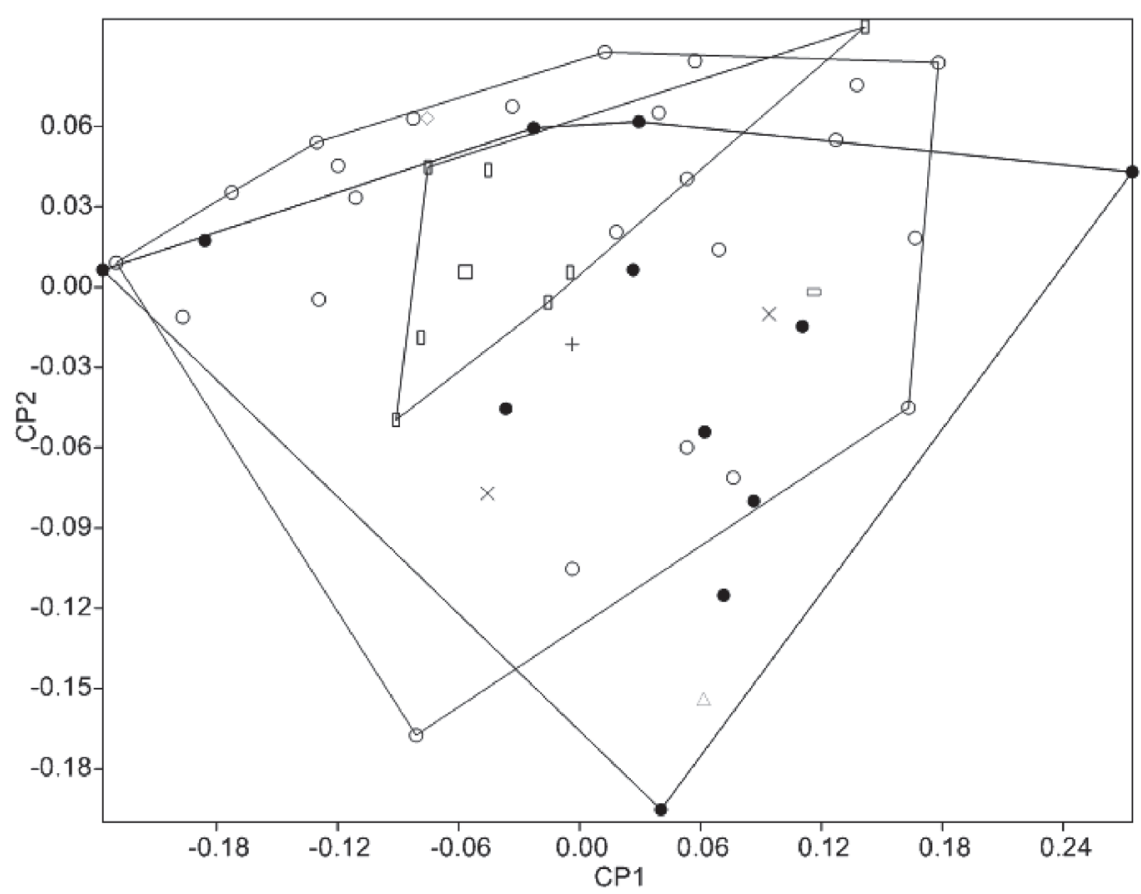

Figura 6. Dispersión morfológica para las distintas categorías de rocas empleadas. Rectángulos: volcanitas básicas. Círculos transparentes: sílex. Círculos rellenos: calcedonias.

Morphological dispersion for the rocks used. Rectangles: basic vulcanites. Empty circles: flint. Filled circles: chalcedony.

hecho no pudo ser comprobado. Es posible, sin embargo, considerar la presencia de un sesgo en la representación de algunas clases de bifaces con muy baja tasa de descarte y/o descartadas en espacios fuera del área de estudio, lo que podría evaluarse nuevamente incrementando el tamaño de los conjuntos estudiados. Otros aspectos vinculados al diseño de los bifaces, como la morfología de las aristas o la simetría relativa, tampoco mostraron diferencias significativas. En conjunto, esto sugeriría una estrategia de reducción similar de las distintas materias primas empleadas.

La cantidad de lascados registrados en las piezas no da cuenta de la existencia de una intención de reducir el espesor de los artefactos por el adelgazamiento bifacial. La presencia predominante de la técnica de reducción bifacial podría estar indicando una falta de intención de conservación de la materia prima y de aprovechamiento al máximo de las rocas. Esto sería indistinto para todos los tipos de rocas analizados, pese a, como se ha mencionado, la representación diferencial de las mismas en las fuentes de rocas del área.
Por medio de los análisis morfométricos realizados solamente en las piezas enteras fue posible resumir gráfica y cuantitativamente el patrón general de variación en los contornos de los artefactos estudiados. Los primeros componentes principales de análisis de Fourier muestran que gran parte de la variación está explicada por la diferenciación entre bifaces y preformas y cabezales líticos propiamente dichos, los cuales se solapan solo parcialmente en el espacio morfológico El primero muestra la tendencia de variación entre formas redondeadas a ovales, mientras que el segundo, contornos triangulares y ovales elongados. Esto puede distinguirse claramente al observar estas dos categorías agrupadas dentro del espacio morfológico (Figuras 3 y 4). Al cruzar la morfología con otras variables técnicas pudimos observar que esta distinción tiende a mantenerse también a lo largo de las rectas de regresión entre forma y cantidad de lascados, y forma y peso. Ambas variables pueden entenderse como un proxy del proceso de reducción en sí mismo, ya que al avanzar este el artefacto incrementa el 
número de negativos de lascado, pierde masa y, en este caso, cambia de forma. En relación con el peso, la dispersión de los residuos, en especial en las piezas más livianas (cabezales líticos), sugiere una amplia variación morfológica para los subproductos finales, independientemente de su masa. Es claro que solo el peso no es un proxy ideal de la morfología, y que otros factores de diseño y performance pueden estar interviniendo en la determinación de la morfología final del cabezal. La amplia variación morfológica en los subproductos finales se suma así a la predominancia de la técnica de reducción bifacial por sobre la de adelgazamiento, lo cual sumaría evidencias para pensar en una falta de estandarización en el proceso general de manufactura de bifaces y cabezales líticos. En cuanto a la relación entre las materias primas y la morfología estudiada de esta manera, observamos un amplio solapamiento que es acorde con lo observado en los test realizados con la totalidad de la muestra (Figura 6), lo cual apoyaría también que incluso considerando solamente las piezas enteras, las rocas fueron usadas indistintamente, pese a su representación diferencial en el espacio.

La variabilidad morfológica detectada entre las rocas volcánicas intermedias-básicas es menor respecto de lo que sucede dentro del grupo de las calcedonias, pese a tener similares pesos, cantidad promedio de lascados y a presentarse en nódulos que, en promedio, pesan menos que los de calcedonia. Esto indicaría una estrategia de reducción más intensiva de las calcedonias para obtener productos finales del mismo peso que los productos finales manufacturados sobre rocas volcánicas intermedias-básicas, hecho que no pudimos corroborar por el promedio de lascados registrado en las piezas, como se ha expuesto más arriba. Sin embargo, al ser este último tipo de rocas menos ubicuo en el ambiente, podría esperarse que hubiesen sido reducidas de forma diferente. Quizás las primeras etapas de reducción se habrían dado en las fuentes, con lo que a los sitios estarían entrando los bifaces ya terminados. Las tendencias registradas llevarían a pensar que las rocas volcánicas intermedias-básicas habrían tenido la calidad necesaria para la manufactura (incluso que hayan sido mejores para la manufactura de puntas de proyectil), y que la calidad superior de calcedonias y sílex no habría sido un factor determinante para la selección de materias primas. Quizás estas últimas habrían sido aprovechadas cuando eran encontradas, pero el aprovisionamiento no estuvo específicamente dirigido a su búsqueda, ni el hecho de que sean más escasas influyó para que fuesen aprovechadas de forma diferencial. No podría afirmarse la mayor o menor conservación de los diferentes artefactos analizados en este trabajo de acuerdo con las materias primas sobre las que fueron manufacturadas. Para evaluar mejor estas tendencias es necesario ampliar la muestra analizada.

Al comparar los resultados de este estudio con aquellos obtenidos para los conjuntos líticos de la costa rionegrina, observamos que las tendencias registradas en la manufactura de bifaces se repiten a nivel de los conjuntos. Es decir, la calcedonia y el sílex fueron las rocas más elegidas también para la talla de otro tipo de instrumentos y esto se detectó para todo el lapso temporal de ocupación de la costa (ca. 6.000-450 años a.p.). A pesar de que las rocas volcánicas son de procedencia local en la costa norte y de calidad menor para la talla, no fueron elegidas mayoritariamente para la fabricación de artefactos de forma expeditiva. En cambio, la calcedonia y el sílex, de presencia mucho menor en las fuentes del área (Favier Dubois y Alberti 2014) y más presentes en la costa oeste (Alberti y Cardillo 2015), fueron rocas elegidas para la manufactura de diferentes clases artefactuales indistintamente (Alberti 2015). Esto indicaría que, más allá del tipo de instrumento manufacturado, lo que primó siempre para la elección de la materia prima fue la calidad de las rocas para la talla, llegando incluso a transportarse nódulos desde y hacia diferentes sectores del espacio.

Tomando en cuenta los resultados de los análisis realizados, podemos delinear algunas tendencias en la muestra analizada. El descarte o abandono de estos bifaces en áreas donde las fuentes de materia prima lítica son ubicuas y abundantes en el ambiente (Favier Dubois y Alberti 2014) estaría contradiciendo la hipótesis del uso de estos artefactos como núcleos, ya que una alta inversión de energía para la conservación de la materia prima no habría sido necesaria entre estos grupos. Sin embargo, las formas más pesadas y redondeadas identificadas en el análisis podrían estar argumentando a favor de que podrían haber funcionado como núcleos o quizás simplemente 
serían los estadios más temprano de la manufactura de bifaces. Así, consideramos que el fin posible de estos artefactos habría sido el de funcionar como herramientas en sí, con la ventaja de ser flexibles y versátiles para la realización de diferentes tareas, o estarían en el proceso de ser formatizados para convertirse finalmente en piezas susceptibles de ser enmangadas. Esto habría satisfecho los requisitos de peso y tamaño que los habrían vuelto herramientas factibles de integrar el toolkit de los grupos cazadores-recolectores del área.

Esta primera aproximación al estudio de la tecnología bifacial permitió delinear algunas tendencias generales de variación en la muestra estudiada. La combinación de técnicas morfométricas con variables tradicionalmente empleadas en el estudio de la reducción bifacial y la serie técnica de este tipo de artefactos (análisis tecnomorfológicos), permitió representar gráficamente las tendencias generales de variación así como la determinación de los factores más relevantes para explicar esta última. Los resultados obtenidos a partir de los análisis morfométricos muestran el potencial de esta técnica para el estudio de la variación morfológica desde un abordaje cuantitativo, ya que permite estudiar a esta como un fenómeno continuo. Esto permite, entre otras cosas, analizar el cambio métrico y morfológico existente entre diferentes categorías como bifaz, preforma o punta de proyectil como parte de una misma trayectoria de reducción o formatización. Por lo demás, las técnicas morfométricas poseen la ventaja que permiten visualizar los cambios ocurridos al ponerlos en relación con una forma media o consenso, y de esta manera extraer las tendencias de variación morfológicas más importantes para el conjunto analizado. En términos comparativos, esto representa una ventaja, ya que las tendencias de variación en distintas muestras pueden ser visualizadas directamente y puestos en relación con factores ambientales, temporales o espaciales. Esta técnica, aplicada por ejemplo al estudio de puntas de proyectil (Cardillo 2005; Castiñeira et al. 2012; Charlin et al. 2014), instrumentos de filo largo (Cardillo et al. 2010), pesas líticas (Scartascini y Cardillo 2009) y raspadores (Cardillo y Charlin 2009), puede ser aplicada sobre diversos materiales para generar y contrastar hipótesis acerca de funcionalidad y cambio tecnológico dentro de los conjuntos de instrumentos, más allá de la forma primaria de los mismos. En el caso particular de la costa rionegrina del golfo San Matías, la ampliación de la muestra estudiada y la división de las piezas de acuerdo con los loci de proveniencia permitirá lograr una aproximación más afinada al estudio de esta tecnología manufacturada y empleada por las sociedades cazadoras-recolectoras que habitaron el área durante el Holoceno Medio y Tardío.

Agradecimientos: este trabajo fue realizado en el marco de los proyectos PIP-CONICET 112-201101-00589 "Ocupaciones tempranas en la costa oeste del golfo San Matías (Río Negro): explorando el inicio del uso intensivo de recursos marinos en Norpatagonia" y PIP-CONICET 112200801-00756 "Paleoambientes, uso del espacio y los recursos en la costa norte del golfo San Matías desde su poblamiento", dirigidos por los Dres. Florencia Borella y Cristian Favier Dubois. A los evaluadores anónimos de este trabajo cuyas sugerencias y comentarios contribuyeron sustancialmente a mejorarlo.

\section{Referencias Citadas}

Alberti, J. 2012. Fuentes de rocas y uso de materias primas líticas en Bahía Final 6, costa norte del golfo San Matías (Río Negro, Argentina). Intersecciones en Antropología 13:237-249.

Alberti, J. 2013. Materias primas líticas y manufactura de instrumentos en tres localidades de la costa oeste del golfo San Matías (provincia de Río Negro, Argentina). Revista Chilena de Antropología 28:79-105.

Alberti, J. 2015. Disponibilidad y Explotación de Fuentes de Materias Primas Líticas durante el Holoceno Medio y Tardío en la Costa del Golfo San Matías, Río Negro, Argentina. Un Enfoque Regional. Tesis Doctoral Inédita. Facultad de Filosofía y Letras, Universidad de Buenos Aires.
Alberti, J. y M. Cardillo 2015. Primary and secondary lithic raw material sources along the western coast of San Matías Gulf (Río Negro province, Argentina): a first approach to their spatial variability. Quaternary Internacional 373:63-71.

Alberti, J. y V. Fernández 2015. Propuesta clasificatoria para las materias primas líticas en Patagonia (Argentina). Arqueología 21:211-233.

Aschero, C. 1975. Ensayo para una clasificación morfológica de artefactos líticos aplicada a estudios tipológicos comparativos. Informe presentado al CONICET. Manuscrito. Buenos Aires.

Aschero, C. 1983. Ensayo para una clasificación morfológica de artefactos líticos. Revisión del año 1975. Informe presentado al CONICET. Manuscrito. Buenos Aires. 
Aschero, C. y S. Hocsman 2004. Revisando cuestiones tipológicas en torno a la clasificación de artefactos bifaciales. En Temas de Arqueología. Análisis Lítico, editado por A. Acosta, D. Loponte y M. Ramos, pp. 7-25. Universidad Nacional de Luján, Luján.

Bleed, P. 1986. The optimal design of hunting weapons: maintainability or reliability. American Antiquity 51:737-747.

Borella, F. 2006. ¿Dónde están los lobos en la costa norpatagónica? Explorando vías para resolver el registro arqueofaunístico. Werken 9:97-114.

Borella, F., M. Cardillo, C. Favier Dubois y J. Alberti 2015. Nuevas investigaciones arqueológicas entre Punta Pórfido y Punta Odriozola: implicancias para el entendimiento de la dinámica de las ocupaciones humanas en la costa oeste del golfo San Matías (Río Negro). Relaciones de la Sociedad Argentina de Antropología XL(1):233-252.

Borella, F., C. Mariano y C. Favier Dubois 2007. Procesos tafonómicos en restos humanos en superficie en la localidad arqueológica de Bajo de la Quinta, Golfo San Matías (Río Negro). En Arqueología de Fuego-Patagonia. Levantando Piedras, Desenterrando Huesos... y Develando Arcanos, editado por F. Morello, M. Martinic, A. Prieto y G. Bahamonde, pp. 403-410. Ediciones CEQUA, Punta Arenas.

Cardillo, M. 2005. Explorando la variación en las morfologías líticas a partir de la técnica de análisis de contornos. El caso de las puntas de proyectil del Holoceno medio-tardío de la Puna de Salta (San Antonio de los Cobres, Argentina). Un enfoque evolutivo. Werken 7:77-88.

Cardillo, M. y J. Charlin 2009. Tendencias observadas en la variabilidad de los raspadores de norte y sur de Patagonia. Explorando interrelaciones entre forma, tamaño e historia de vida. Segundo Congreso Argentino y Primero Latinoamericano. Arqueometría Latinoamericana Vol. 2, pp. 351-359.

Cardillo, M., J. Charlin y K. Borrazzo 2010. Una exploración de la variación métrica y morfológica en instrumentos de filo largo en Patagonia meridional. En La Arqueometría en Argentina y Latinoamérica, editado por S. Bertolino, G.R Cattaneo y A.D. Izeta, pp. 147-152. Editorial de la FFyH, Universidad Nacional de Córdoba, Córdoba.

Castiñeira, C., J. Charlin, M. Cardillo y J. Baeza 2012. Exploring morphometric variations in Fishtail projectile points from Uruguay, Pampa and Patagonia. En Current Research in the Pleistocene. Special Edition. Southbound: Late Pleistocene Peopling of Latin America, editado por L. Miotti, M. Salemme, N. Flegenheimer y T. Göebel, pp. 57-61. Tops Printing Inc., Texas.

Charlin, J., M. Cardillo y K. Borrazzo 2014. Spatial Patterns in Late Holocene Lithic Projectile point Technology of Tierra del Fuego (Southern South America): assessing size and shape changes. World Archaeology 46:78-100.

Favier Doubois, C. y J. Alberti 2014. Materias primas líticas en la costa norte del golfo San Matías (Río Negro, Argentina): distribución de fuentes y tendencias generales en su aprovechamiento. Revista del Museo de Antropología 7(1):93-104.

Favier Doubois, C. y F. Borella 2011. Contrastes en la costa del golfo: una aproximación al estudio del uso humano del litoral rionegrino. En Arqueología de Pescadores y Marisqueadores en Nordpatagonia. Descifrando un Registro de más de 6.000 Años, editado por F. Borella y M. Cardillo, pp. 13-42. Editorial Dunken, Buenos Aires.

Favier Dubois, C., F. Borella y R. Tykot 2009. Explorando tendencias en el uso humano del espacio y los recursos en el litoral rionegrino (Argentina) durante el Holoceno medio y tardío. En Arqueología de la Patagonia - Una Mirada Desde el Último Confín, editado por M. Salemme, F. Santiago, M. Álvarez, E. Piana, M. Vázquez y E. Mansur, pp. 985-997. Editorial Utopías, Ushuaia.

Favier Dubois, C., C. Stern y M. Cardillo 2009. Primera caracterización de los tipos de obsidiana presentes en la costa rionegrina. En Arqueología de la Patagonia - Una Mirada desde el Último Confín, editado por M. Salemme, F. Santiago, M. Álvarez, E. Piana, M. Vázquez y E. Mansur, pp. 349-359. Editorial Utopías, Ushuaia.

Fishman, G. 1995. Monte Carlo: Concepts, Algorithms, and Applications. Springer, New York.

Gero, J. y J. Mazzullo 1984. Analysis of artifact shape using Fourier Series in closed form. Journal of Field Archaeology 11:315-322.

González Díaz, E. y E. Malagnino 1984. Geomorfología de la provincia de Río Negro. Actas del IX Congreso Geológico Argentino, pp. 1-159. Secretaría de Minería, Buenos Aires.

Hammer, Ø., D. Harper y P. Ryan 2001. PAST. Palaeontological Statistics software package for education and data analysis. Palaeontologia Electronica 4:1-9.

Hocsman, S. y P. Escola 2006-2007. Inversión de trabajo y diseño en contextos líticos agro-pastoriles (Antofagasta de la Sierra, Catamarca). Cuadernos del Instituto Nacional de Antropología y Pensamiento Latinoamericano 21:75-90.

Kelly, R. 1988. The three sides of a biface. American Antiquity 53:717-734.

Kuhl, F. y C. Giardina 1982. Elliptic Fourier features of a closed contour. Computer Graphics and Image Processing 18:236:258.

Kuhn, S. 1994. A formal approach to the design and assembly of mobile toolkits. American Antiquity 59:426-442.

Nami, H. 1988. Arqueología experimental, tecnología, artefactos bifaciales y modelos. Estado actual del conocimiento en Patagonia y Tierra del Fuego. Anales del Instituto de la Patagonia 18:157-176.

Nelson, M. 1991.The study of technological organization. Archaeological Method and Theory 3:57-100.

R Development Core Team 2009. R: A language and environment for statistical computing. Vienna, Austria: R Foundation for Statistical Computing. http://www.R-project.org (15 agosto 2014).

Rohlf, F. 2006. TPS serie softwares. http//life.bio.sunysb.edu/ Corp (20 agosto 2014).

Scartascini, F. y M. Cardillo 2009. Explorando la variabilidad métrica y morfológica de las "pesas líticas" recuperadas en el sector norte de la costa del golfo de San Matías. Segundo Congreso Argentino y Primero Latinoamericano. Arqueometría Latinoamericana Vol 1, pp. 162-168. 